\title{
SOFIA GALLARDO*
}

Foucault y la Ideología

\begin{abstract}
A primera vista podría sorprender la intención de rastrear el A término de ideología en la obra de Michel Foucault, si consideramos que él renuncia sin ambigüedades a esta noción por creer que cristaliza una imposición metafísica de las formas tradicionales de historiar la historia. Sin embargo, la relevancia del tema está precisamente en la deconstrucción - a veces implícita - que hace del concepto de ideología, como premisa necesaria para la construcción de los supuestos fundamentales de su pensamiento.

Es importante aclarar de entrada que este problema no es central en la investigación foucaultiana, sino que está contenido en la crítica sistemática a la historia global, al sujeto fundante y a la díada ciencia-ideología. De hecho, las alusiones al término de ideología aparecen como corolario de estudios más sustantivos, son sistemáticas y contundentes a pesar de su brevedad, e inducen a replantear el problema en función del discurso la primacía del poder.

El lector minucioso encontrará dos apreciaciones diferentes de ideología en su obra. Una de carácter positiva, en la que se identifica al concepto con una práctica discursiva circunscrita en un ámbito del saber y de la sociedad, que es ajeno al interés de su quehacer arqueológico y genealógico. La otra consideración está marcada por un fuerte desdén y se refiere a la ideología como noción eterna inserta en una concepción de la historia fundada en totalizaciones y continuidades. Esta acepción opera a manera de obstáculo a su proyecto teórico, razón que explica el por qué de su apartamiento y deconstrucción.

En principio, Foucault define a la ideología - en su segunda connotación - como un ídolo y un prejuicio, y se da a la tarea de

- Centro de Investigación y Docencia Económica
\end{abstract}


destruirla poniendo en práctica la metáfora nietzscheana de filosofar con el martillo. De aquí deriva su método, definido por Franco Re$1 \mathrm{ll}^{1}$ como el dispositivo foucault, que concibe a la teoría como caja de herramientas. Su objetivo es proponer instrumentos de descripción y análisis en vez de sistemas, y enmarcarlos en una reflexión histórica sobre situaciones dadas. Para ello, busca demoler las grandes síntesis que omniexplican el orden burgués - por medio de astucias metafísicas - para apartar su discurso de la "tentación ideológica".

De esta manera, su obra se aparta del tema de las ideologías y no ofrece ideología, sino herramientas para teorizar las luchas específicas (antipsiquiátrica, anticarcelaria, ..) y poder vincularlas entre sí por medio de una estrategia teórica. ${ }^{3}$

El método foucaultiano hace suyos los procedimientos del positivismo, que consisten en un seguimiento crítico de la evolución de los conceptos y del pensamiento, en los documentos que constituyen los diferentes estados de los saberes. De otra parte, sin embargo, se halla imbuido por un profundo nihilismo - al que no escapa su generación-derivado de la enunciación nietzscheana del fracaso del postulado teórico del positivismo: "no hay hechos sólo interpretaciones". Domina la convicción de que los hechos en sí mismos no significan nada y que con los mismos datos podrían construirse diferentes narraciones. $\mathrm{Y}$ es precisamente con el uso del dispositivo foucault como destruye y reconstruye interpretaciones en el marco de una historia de las rupturas y discontinuidades.

Cuando Foucault escribió sus primeras obras, el ambiente intelectual que lo rodeaba estaba saturado por el tema de la ideología. Se le ubicó en teorías omnicomprensivas que giran en torno a las formaciones sociales, los sistemas semióticos o la weltanschauung. Se aspiró a la formulación de una teoría general de las ideologías, pero a medida que se pretendió otorgarle un sustrato científico se estrelló frente a la contradicción entre su dimensión abstracto-universal y su especificidad de concreción histórica. Así, al estiramiento teórico como aglutinador de todo tipo de sistemas de

1 Franco Rella El dispositivo Foucauh, Venezia, 1977.

2 Michel Foucault "Poder y Estrategia" en Microffsica del poder, Las ediciones de La Piqueta, Madrid, 1980, p. 164.

3 Michel Morey prólogo a Sero, poder, verdad, Conversaciones con Michel Foucault, Editorial Materiales, Barcelona, 1978, p.14. 
representación (creencias, mitos, conceptos...), seguía el encogimiento para explicar sus características político-sociales. No se llegaba a abarcar el fenómeno global ni a matizar suficientemente la particularidad de los acontecimientos.

En 1968, Althusser se erige en autoridad del tema en Francia, con su trabajo "Ideología y Aparatos Ideológicos de Estado". Se sirve de la aproximación lacaniana a la teoría de Freud y se deslinda de las concepciones marxistas que consideran a la ideología como una instancia superestructural que es determinada unívocamente por la base económica, sin por esto lograr trascender una concepción totalizadora de la historia. Parte de la tesis de que la ideología es eterna - no tiene historia - y por tanto contiene siempre la misma estructura formal, donde el término central y decisivo es el sujeto. Su objetivo es analizar la ideología por oposición a la ciencia como una categoría funcional que, al crear la ilusión del sujeto como portador del sentido y constituir a los individuos en sujetos, incide en las relaciones de producción creando efectos de dominación (explotación) sobre la clase obrera.

En este contexto, Foucault se aparta del término e ideología. Empieza por hurgar en sus condiciones de posibilidad y detiene su atención en la historia tradicional, en la historia de las ciencias y en un cierto tipo de marxismo. El autor manifiesta con precisión y contundencia sus inconvenientes en la siguiente referencia textual de "Verdad y poder".

La noción de ideología me parece difícilmente utilizable por tres razones. La primera es que, se quiera o no, está siempre en oposición virtual a algo que sería la verdad. Ahora bien, yo creo que el problema no está en hacer la partición entre lo que, en un discurso, evidencía la cientificidad y la verdad y lo que evidencia otra cosa, sino ver históricamente cómo se producen los efectos de verdad en el interior de los discursos que no son en sí mismos ni verdaderos ni falsos. Segundo inconveniente, es que se refiere, pienso, necesariamente a algo como a un sujeto. Y tercero, la ideología está en posición secundaria respecto a algo que debe funcionar para ella como infraestructura o determinante económico, material, etc. Por estas tres razones, creo que es una noción que no puede ser utilizada sin precaución. ${ }^{4}$

4 Michel Foucault "Verdad y poder", en Microfisica del poder, op. cit, pp. 181-182. 
Estas objeciones llevan implícita la deconstrucción de tres oposiciones binarias: ideología e historia, ideología y práctica, e ideología y ciencia. Michel Foucault niega el carácter esencial, constituyente y determinante de la conciencia humana sobre el devenir, el hacer o el sentido. Y, de hecho, propone una inversión logocéntrica en la que el saber y el poder operan como semitrascendentes sobre una noción de ideología subordinada y limitada a la existencia de práctica discursiva entre otras prácticas o de estrategia extrema de poder.

\section{Historia e historiografia}

El significado del concepto de ideología varía necesariamente según se encuentre comprendido en las diferentes concepciones sobre la historia. Foucault habla a través de una mutación epistemológica de la historia que aún no ha terminado y que se caracteriza por una ardua crítica a la historia continua y a la historia marxista, así como por un esfuerzo importante de descentramiento de la historia de las ciencias, que le permite avanzar en la construcción del método de una historia nueva. Esta ruptura con las interpretaciones anteriores conlleva la desarticulación de los diversos usos de la ideología que abrigan. Precisar sus desemejanzas es fundamental para matizar el cuestionamiento a las distintas connotaciones de la ideología.

En La arqueologia del saber, Foucault identifica a la historia continua o global como "uso ideológico de la historia". Se trata de una función conservadora que asume a las continuidades como el fundamento de toda historicidad posible y que se resiste al uso manifiesto de las categorías de ruptura y diferencia.

Para ahondar en las características de esta forma tradicional de historia, el autor hace una búsqueda minuciosa de sus condiciones de posibilidad. Reconoce una filosofía de la historia fundada en una racionalidad de la teleología del devenir, en la relatividad del saber histórico y en la posibilidad de descubrir o constituir un sentido a los acontecimientos. Encuentra una historia del pensamiento sustentada en el ejercicio fundador del sujeto - de todo devenir y de toda práctica - y su tendencia a reintegrar las diferencias, bajo la modalidad de conciencia histórica. Identifica una antropologización 
de Marx como historiador de las totalidades culturales y portador tiel humanismo, así como una interpretación de Nietzsche que le atribuye la paternidad de una filosofía trascendental y la investigación de lo primigenio. Y, por último, registra una negación del estructuralismo por su imposibilidad de explicar el devenir, ya que se asume una oposición irreductible entre estructura e historia.

De esta manera, Foucault examina desde sus fundamentos a la historia global. La describe como una historia que organiza los fenómenos alrededor de un centro único, llámese significación, espíritu de una época o visión del mundo; como un sistema de relaciones homogéneas sustentado en la causalidad y la analogía; y como articulada en un principio de cohesión de grandes períodos o totalizaciones, que se explican por la acción sintética del sujeto y por la lógica de las continuidades. Estas características de la historia global definen, asimismo, los atributos de la ideología en tanto que práctica discursiva que se ocupa de la historia.

El proyecto foucaultiano tiene por objeto invalidar esta historia continua y deslindarse del "uso ideológico de la historia", al poner en práctica su método arqueológico que consiste en la "descripción intrínseca del monumento", 6 esto es de sus condiciones de posibilidad.

Recordemos que cuando Foucault hace la arqueología de la historia global se refiere también a una cierta historia marxista, que parte de una interpretación de Marx como historiador de las totalidades culturales. Bajo esta concepción, nos dice, "la ideología está en posición secundaria respecto a algo que debe funcionar para ella como infraestructura o determinante económico, material, etc." ${ }^{7}$

El autor rebate el supuesto marxista sobre el que se constituye esta noción de ideología, a saber, el de la formación social como la articulación entre dos instancias diferenciadas: infraestructura y superestructura. Afirma que se trata de una explicación monocausal de la historia que parte de la totalización llevada a cabo por la determinación económica en última instancia. Foucault contrapone una interpretación multicausal, donde la formación social constituye un espacio correlativo para la arqueología, ya que es el campo en que emergen los objetos discursivos y las condiciones de apropiación de

6 Jdem, p.11

7 "Vcrdad y poder", op. cit, pp. 181-182. 
los discursos. ${ }^{8}$ De otra parte, piensa en términos de discursos y no de ideología, y considera en su genealogía que los discursos son de por sí poderes y no necesitan encontrar su fuerza material en el modo de producción. ${ }^{9}$

Cabe señalar que, si bien Foucault busca estudiar las formaciones sociales en su dispersión - a través de la producción de las diferentes prácticas discursivas - y objeta el uso de la ideología como infraestructuralmente determinada, esto no significa que invalide el análisis clasista del marxismo.

Paralelamente a estas modalidades de historia, el autor identifica a la historia de las ciencias, de las ideas, de la filosofía, de la literatura y demás, en cuyas preocupaciones se reconoce ya que están orientadas a los fenómenos de la ruptura y de las discontinuidades. Hace mención de Bachelard, Canguilhem, Guéroult y Althusser, quienes reivindican los recortes y los límites frente a la tradición, y las transformaciones ante las continuidades. Asimismo, coincide con ellos cuando plantean un nuevo tiempo y racionalidad diferente a la búsqueda de los origenes; y cuando explicitan principios de su metodología, tales como la coherencia interna de los documentos y las reglas de constitución y validez de los conceptos.

No obstante, se aparta de la historia de las ciencias porque ésta se sitúa en el umbral de la cientificidad, ahí donde la figura epistemológica responde a ciertas leyes de construcción de las proposiciones. Esta concepción tiene como premisa la oposición y triunfo de la ciencia frente a la ideología. Busca dar respuesta a cómo una ciencia se ha establecido por encima y contra un nivel precientífico que a la vez la preparaba y la resistía de antemano, cómo ha podido franquear los obstáculos y las limitaciones que seguían oponiéndose a ella. 10

Foucault trabajó en una historia arqueológica, que toma como referencia al umbral de epistemologización pero apoyándose en el umbral de la positividad. Busca descubrir cómo las prácticas discursivas dan lugar a un saber y cómo ese saber adquiere el estatuto de ciencia, ideología, literatura y otras manifestaciones discursivas. De esta manera, se refuta tanto la idea de que la ciencia se desarrolla a

8 La arqueologia del saber, op. cit, p. 348.

9 Mark Poster, Foucault, manxismo e historia, Paidós Studio 64, Argentina, 1987, p. 124.

10Michel Foucault, . La arqueologfa del saber, op. cit, p.320. 
expensas de la ideología como el modelo de una ideología que tiene una existencia epistemológicamente negativa, dependiente y previa a la ciencia.

Además planteó que aun cuando el análisis arqueológico es útil para la descripción histórica de los distintos saberes, no se agota en las figuras epistemológicas y en las ciencias, sino que puede desarrollarse en direcciones diferentes. Por ejemplo, considera el proyecto de descripción arqueológica sobre la sexualidad, un cuadro y la política.

El proyecto arqueológico está ubicado en la actual mutación epistemológica de la historia y protagoniza un triple descentramiento. Niega las interpretaciones que aluden a una teoría marxista antropologizada y rescata el análisis histórico de las relaciones de producción y de la lucha de clases de Marx. Asimismo, desconoce la exégesis de una teoría nietzscheana trascendentalista y redimensionaliza a la genealogía de Nietzsche en abierta oposición a la búsqueda del origen, al despliegue metahistórico de las significaciones ideales y de los indefinidos teleológicos. Por último, admite la importancia del estructuralismo por haber descentrado al sujeto. Sin embargo, no deja de reconocer las limitaciones de la oposición estructura-devenir y el alcance parcial de los problemas que plantea el estructuralismo, que están lejos de cubrir el campo metodológico de la historia. ${ }^{11}$

Así, en su fase arqueológica, Foucault hace una historia de la ruptura a la vez que contribuye a la ruptura de los fundamentos de la historia global. Asume la discontinuidad como instrumento y objeto de investigación para desprenderse del uso ideológico de la historia e inscribirse en la práctica discursiva de los saberes.

\section{Sujeto e ideologia}

Michel Foucault se aparta de la noción de ideología porque piensa que se refiere necesariamente a algo como a un sujeto. ${ }^{12}$ Es, por tanto, consecuente con una de las grandes obsesiones de su obra, que es lograr el descentramiento del sujeto. En este esfuerzo, desarrolla una interpretación de la historia como un "proceso sin sujeto, ni fi- 
nes", describe a las ideas en el ámbito del discurso por considerar que su inteligibilidad no procede de los sujetos y, finalmente, estudia al poder como estrategia en vez de atributo del Sujeto. De tal suerte, invalida el término de ideología porque autentifica al Sujeto como fundante de la historia, del saber y del poder, como originario de todo hacer y de todo decir.

La preocupación foucaultiana por negar al sujeto constituyente y desantropologizar al saber, está influida por la aproximación estructuralista al lenguaje. Ésta analiza a la lengua como un sistema de signos - considerado en sí y por sí mismo-, que produce significaciones a través de las relaciones de diferencia entre los elementos linguísticos, sin la intervención fundadora de un sujeto. Si bien Foucault intenta deslindarse de las explicaciones sistémicas, retoma del estructuralismo el apartamiento del sujeto para pensar el saber. Asimismo, aplica este principio a las relaciones de poder cuando las define como "intencionales y no subjetivas"; esto es, como "grandes estrategias anónimas" cuya racionalidad está en las tácticas, que al interrelacionarse constituyen dispositivos de conjunto. ${ }^{13}$

Hasta 1968, Foucault va a sostener una concepción antisubjetivista que se manifiesta en una oposición contundente a todas las filosofías de la conciencia y, por tanto, al humanismo incluyendo al existencialismo sartreano.

En las palabras y las cosas niega de hecho la noción de ideología cuando sustenta la tesis de que los sujetos no son eternos ni constituyentes porque no son la fuente de ias ideas, sino que están históricamente constituidos. De aquí, que su objetivo sea definir la fecha de nacimiento del hombre moderno a tráves de la descripción de los cambios en las reglas de formación de los discursos y, en concreto, del desentrañamiento de la emergencia de las ciencias humanas. Concibe al hombre moderno como un nudo epistemológico por ser a la vez el objeto y el sujeto de su conocimiento. Y, a partir de este círculo hermenéutico, se propone descifrar los mecanismos con los cuales las ciencias humanas llegan a dominar -en vez de liberaral sujeto. Esto es, se refiere a la entronización de la vida, el trabajo y el lenguaje como semitrascendentes del conocimiento, que culminaron el proceso de antropologización del saber.

13 Historia de la scrualidad, I. La voluntad de saber, Siglo XXI editores, México, 1981, pp. 115-116. 
Su preocupación va a ser desenmascarar esta süjeciốn del sujeto y analizar las condiciones de posibilidad de la muerte epistemologica del hombre, es decir, de la desantropologización del saber que lo constituye. Con este propósito se da a la tarea de interrogar los límites del pensamiento y de reanudar así el proyecto nietzscheano de la crítica general de la razón. ${ }^{14}$

Foucault avanza en la definición de este problema en La arqueologia del saber a través del perfeccionamiento y explicitación de sus recursos metodológicos. Afirma que no ha querido excluir el problema del sujeto al invalidar el carácter originario que le atribuye la noción de ideología, sino definir las posiciones y las funciones que el sujeto podrá ocupar en la diversidad de los discursos. Y a fin de desatar las últimas sujeciones antropológicas o, de manera más precisa, evidenciar cómo pudieron formarse esas sujeciones, ${ }^{15}$ devela un nuevo dominio de análisis: el de las modalidades enunciativas y las prácticas discursivas.

Se da a la búsqueda de la ley de las diversas enunciaciones, a:í como del lugar de donde vienen. Encuentra que los planos desde los que se habla son discontinuos y están unidos por todo un haż de relaciones establecido por las prácticas discursivas, que permiten la renovación de las modalidades enunciativas. Queda excluida, por tanto, la idea de una conciencia previa a toda palabra. Lo que interesa es detectar un campo de regularidad para diversas posicionés de subjetividad (no como la expresión del sujeto), desde donde pueda determinarse la dispersión del sujeto y su discontinuidad consigo mismo.

Puede observarse que, si bien Foucault no excluye la cuestión del sujeto, sí es contundente cuando retira al discurso el derecho exclusivo e instantáneo a la soberanía del sujeto. ${ }^{16}$ Niega así el principio de toda ideología que ve a los sujetos como la conciencia que se expresa en los discursos, para recuperarlos en su dispersión a tráves de un conjunto de reglas anónimas que los forman.

El autor aplica todos estos supuestos metodológicos en la construcción de los objetos de su trilogía arqueológica: la locura, la enfermedad y las ciencias humanas. Aun cuando los dos primeros reciben un tratamiento epistemológico clásico (en el que todo cono- 
cimiento se elabora en función de una urgencia práctica) y el tercero un procedimiento epistemológico nuevo (donde todo conocimiento se elabora porque otros conocimientos le dieron la posibilidad de aparecer), los tres objetos son analizados con reglas anónimas que constituyen y subordinan a los sujetos en su dispersión: los locos, los enfermos y el hombre moderno.

Aún más, su posición antisubjetivista se ratifica al excluir a la ideología de entre las cinco condiciones de posibilidad con que clasifica la formación de los saberes: infraestructural, institucional, discursivo, perceptivo y referencial.

Pasado el apogeo estructuralista, a fines de la década de los sesenta, Foucault aminora su tendencia inicial de relegar el tema del sujeto, ya que advierte que sin alguna teoría del sujeto no es posible analizar efectos de poder con la resistencia a la autoridad. Sin embargo, consecuentemente con las premisas de su pensamiento y con el propósito de atenuar el riesgo de sucumbir a la metafísica, continua negando la categoria de sujeto fundante y trascendente para anteponer la dispersión de los sujetos constituidos y sus relaciones atravesadas por una amplia red de saberes y poderes.

En esta segunda fase, privilegia cuestiones que subyacían latentes. Deconstruye la díada saber-poder en la que el papel dominante del saber casi había sofocado el tema del poder. De esta manera, hace una inversión logocéntrica que otorga primacía al poder sin menoscabo del saber, ya que se autoimplican y son inseparables. Amplía el dispositivo arqueológico como genealogía y se desentiende de la máscara epistemológica para concentrarse en la política del saber. Y, por último, retoma de la arqueología el discurso en su materialidad de acontecimiento enunciativo e introduce el poder, en tanto que condición de posibilidad que ha hecho invisible este "dominio transparente".

En El orden del discurso la preocupación deja de ser, en primer término, invalidar al sujeto como creador del saber y dador del sentido, para negarlo como voluntad que posee el poder de determinar el control, la selección y redistribución de los discursos. En consecuencia desconoce el supuesto de que la ideología de la clase dominante expresa la representación del mundo en cada época y se avoca al estudio de la "imposición" de reglas anónimas que constituyen la política del saber. Hasta aquí, priva una concepción negativa del poder en la obra de Foucault. 
Ya en Vigilar y castigar analiza la transformación de los sujetos a través de una tecnología disciplinaria o modalidad positiva del poder. Y, en La voluntad de saber va más lejos aún al encarar la confesión como un medio de autentificación subjetiva y autoproducción de sujetos, e interpreta una práctica discursiva determinada no por la inhibición sino por la incitación. Aquí introduce el tema del placer vinculado al poder y al saber. Por último, y en la misma línea, en El uso de los placeres; La inquietud de sí; y Los testimonios de la car$n e$, se propone estudiar prácticas discursivas que dan cuenta de la genealogía del hombre de deseo.

En la fase genealógica, los condicionantes del sujeto y el apartamiento de la noción de ideología están implícitos en la renuncia de los cinco postulados marxistas sobre el poder. ${ }^{17}$ Consideramos importante citar estos condicionantes porque destruyen la pretensión soberana del sujeto sobre el poder y lo definen como una estrategia. Veamos:

- el sujeto (la clase dominante) no posee el poder, lo ejerce;

- el poder no se localiza en un sujeto (institución estatal), es una microfísica;

- el poder no es pura superestructura determinada, constituye y atraviesa con múltiples redes al sujeto (cuerpo social);

- la represión y la ideología son estrategias extremas de poder, éste produce lo real a través de una trasformación técnica de los cuerpos que tiene el efecto de normalización; y- el poder no está depositado en un sujeto (clase o polo dominante) de manera unilateral, hay solamente relaciones de poder con resistencias múltiples.

Se aprecia cómo Michel Foucault refuta el carácter absoluto del sujeto, a la vez que le otorga una condición trascendente al poder. Con esto, desmantela el sustrato metafísico de la ideología que define a la conciencia humana como el sujeto originario de todo sentido y de todo devenir y práctica; para erigir la primacía de la práctica discursiva y del poder como categorías descriptivas y analíticas 
que son funcionales a su genealogía (por estar exentas de la subordinación antropológica).

\section{Ciencia e ideología}

El proceso de desconstrucción foucaultiano de la noción de ideología conlleva el cuestionamiento de las ideas de totalización y de sujeto constituyente, y culmina en la desarticulación de su tercer y último fundamęnto teórico, que la define negativamente por oposición a la ciencia. Para estos efectos, el autor reflexiona desde los niveles epistemológico, arqueológico y genealógico.

- En la historia de las ideas, la dualidad ciencia-ideología deriva de una relación de inclusión y exclusión del eje epistemológico: conciencia-conocimiento-ciencia. El primer eslabón se identifica con el Sujeto e imprime, necesariamente, una cierta carga de subjetividad al conjunto. Por tanto, el punto de equilibrio de este eje - el conocimiento - plantea una interrogante trascendente, porque opera como síntesis constituyente. Y la ciencia forma el polo positivo que mantiene un estado de inclusión en el eje epistemológico. En contraposición, la ideología deviene en el polo negativo y excluido, que se inscribe en la relación: inconsciencia-desconocimiento-ideología.

A este nivel, la contradicción entre ciencia e ideología se explica a través de la pureza o impureza de su esencia, de su utilización técnica como instrumento político en una sociedad y de la conciencia de los sujetos que la constituyen. En concreto, estas características se manifiestan en la connotación peyorativa que el marxismo le ha dado al término de ideología frente al de ciencia. La mención es especialmente válida para Marx y el primer Althusser.

Según estas aproximaciones teóricas, la oposición binaria ciencia-ideología corresponde más a una cuestión de naturaleza que de funcionamiento; ya que la ciencia es portadora de lo verdadero y lo puro; mientras que la ideología vehiculiza lo falso y lo impuro. Sin embargo, la preocupación de fondo está en la identificación de estos supuestos con la historia, que se traduce en la denuncia de un régimen de dominio capitalista vinculado con la mentira y una aspiración comunista de libertad asociada con la verdad.

Esta aproximación funda la relación entre ciencia e ideología sobre los argumentos que Michel Foucault pretende desmontar. 
Esto es, el atributo universal, eterno y absoluto de la verdad y la mentira, el centramiento en una clase social, así como la interpretación de los modos de producción como totalizaciones. Lo que hace el autor es remitir la generación de estos problemas a las connotaciones subjetivistas y trascendentes del eje epistemológico.

Es comprensible por tanto que Foucault intente resolver los equívocos de esta contradicción - ciencia vs. ideologra - apartándose del nivel epistemológico. Para estos efectos se inscribe en el nivel arqueológico y propone un nuevo eje: práctica discursiva-saber-ciencia. Elimina del primer término de la relación la idea de la conciencia anterior a toda palabra y define a la práctica discursiva como un conjunto de condiciones de ejercicio de la función enunciativa. Asimismo, en el punto de equilibrio de la relación, el sujeto aparece situado y dependiente. El autor asocia al saber con to previo, pero se cuida de enfatizar que no se trata de un dato, experiencia, preconocimiento o estadio arcaico. Lo concibe como un "conjunto de elementos formados de manera regular por una práctica discursiva y que son indispensables a la constitución de una ciencia, aunque no estén necesariamente destinados a darle lugar". ${ }^{18}$ En el ámbito del saber sólo pertenecen a un domino de cientificidad las proposiciones que obedecen a ciertas leyes de construcción y coexistencia con otras prácticas discursivas de índole económica, política, ideológica, artística y demás.

Es claro que para Foucault el saber no es un equivalente de la ideología, es un espacio aún más amplio que no resulta de la exclusión y descalificación de los elementos que no se articulan en la ciencia y que todavía no han sido conquistados por ella. Por tanto, la ciencia y la ideología son prácticas discursivas que funcionan entre otras prácticas y que se articulan alli donde se perfilan sobre el saber, sin identificarse con él, borrarlo o excluirlo.

A manera de conclusiones generales sobre la descripción arqueológica de la relación ciencia-ideología, el autor formula las siguientes proposiciones:

- el que algunos discursos científicos abran espacio a la ideología, no es razón suficiente para descalificar el conjunto de sus enunciados; 
- el funcionamiento ideológico de una ciencia (contradicciones, lagunas e inconsistencias teóricas) debe analizarse al nivel de la positividad y de las relaciones entre las reglas de formación y las estructuras de la cientificidad;

- el papel de la ideología no disminuye a medida que crece el rigor y se disipa la falsedad del discurso; y

- ocuparse del funcionamiento ideológico de una ciencia es ponerla en discusión como formación discursiva. ${ }^{19}$

El límite de esta propuesta está, según lo advierte el propio Foucault, en no haber determinado el funcionamiento y la distribución del poder y su vinculación con el saber. En consecuencia, plantea como nueva alternativa el método genealógico. Retoma al discurso en su materialidad de acontecimiento enunciativo, tal como lo concibió en la arqueología; pero para entender cómo este "dominio transparente" se ha hecho invisible, se coloca a nivel de sus condiciones de posibilidad e introduce la idea de poder.

El discurso adquiere el estatuto de acto político, porque es un poder de control-producción sobre los discursos lo que origina las serializaciones de acontecimientos discursivos en los que nos reconocemos. Ya no se trata únicamente del saber sino de la política del saber, de las relaciones entre saber y poder, y por tanto de la política del discurso. ${ }^{20}$

A partir de las consideraciones anteriores, es posible analizar la propuesta foucaultiana frente a la primera objeción que planteó a la noción de ideología en "Verdad y poder".

...se quiera o no, está siempre en oposición virtual a algo que sería la verdad. Ahora bien, yo creo que el problema no está en ha- cer la partición entre lo que, en un discurso, evidencia la cientificidad y la verdad y lo que evidencia otra cosa, sino ver históricamente cómo se producen los efectos de poder en el interior de los discursos que no son en sí mismos ni verdaderos ni falsos. ${ }^{21}$

19. Ibidem, pp. 312-313.

20 El orden del discurso, Cuadernos Marginales 36, Tusquets editores, España, 1987.

21"Verdad y poder", op. cit, pp. 181-182. 
Foucault adopta una aproximación de corte nietzscheano. Sustenta que todos los discursos son meras posibilidades y que su valor reside en el papel que desempeñan en la constitución de las prácticas, como portador de saberes y poderes. De tal suerte, atribuye un valor extra epistemológico a los discursos, que les niega una connotación verdadera, científica o ideológica, en sí misma. Lo importante son los efectos propios de lo; discursos concebidos socialmente como verdaderos, esto es, la política de los discursos.

$\mathrm{Su}$ alternativa es radical: dejemos de pensar en términos de ciencia-ideología para reflexionar en función de verdad-poder. Este binomio significa que no hay verdad fuera del poder ni los efectos de poder se dan desligados de la verdad $\mathrm{y}$, aun más, que la verdad tiene una existencia histórica y posee efectos reglamentados de poder. Así, cada sociedad tiene un régimen de verdad que define a los discursos como verdaderos, que determina su producción y establece sus divergencias con los discursos socialmente falsos, así como el estatuto de quienes los sancionan.

En las sociedades occidentales u occidentalizadas, la verdad se identifica con el discurso científico y con aquellas instituciones económicas y políticas que la producen, transmiten y requieren. $\mathrm{La}$ verdad constituye, también, el núcleo de luchas ideológicas que se manifiestan en el debate político y en el enfrentamiento social.

En medio de todo esto, Foucault atribuye al intelectual la función de analizar los efectos de poder de los discursos verdaderos, y, sólo de manera secundaria, preocuparse si un discurso vehicula o no una ideología científica. Su problema político esencial no debe ser, por tanto, criticar los contenidos ideológicos ligados a la ciencia, hacer que su práctica cientifica esté acompañada de una ideología justa o "cambiar la conciencia" de la gente. La cuestión es modificar el régimen político, económico e institucional de la producción de la verdad.

Las consecuencias de estas reflexiones genealógicas representan un gran avance frente a la historia de las ideas y a su propia arqueología, porque niegan el valor absoluto y universal de la verdad científica por oposición a la impureza teórica de la ideología, a la vez que circunscriben lo verdadero a sus determinantes sociales y lo analizan en su perspectiva histórica a partir de los impactos políticos que genera. En rigor, Foucault no niega ni descalifica a la ideología, la admite en tanto que existe, aunque no constituya el eje de sus 
preocupaciones ni el campo de análisis de sus investigaciones. Le interesa la cuestión política - que no es el error, la ilusión, la conciencia alienada o la ideología - , que es la verdad misma. ${ }^{22}$

\section{Consideraciones finales}

La ideología, que es un tema marginal en la obra de Michel Foucault, ha sido un buen pretexto para reconstruir los ejes principales de su filosofía. Con este objetivo, nos hemos referido a la crítica foucaultiana de la historia tradicional, de la historia de las ideas y de un cierto marxismo, a través de la negación de tres de sus tesis centrales: la historia continua, el sujeto fundante y el dominio de la ciencia. Lo relevante es que estas tesis constituyen precisamente las objeciones más importantes que el autor esgrime en contra de la noción de ideología.

Foucault se aparta del nivel epistemológico de análisis que fundamenta las concepciones anteriores y propone el método arqueológico para identificar a la ideología en el ámbito del saber como práctica discursiva entre otras prácticas. Sin embargo, posteriormente abandona la primacía de esta descripción de las condiciones de posibilidad de los objetos de discurso, para hacer genealogía. Pasa a centrarse en el poder como instancia trascendente asociada al saber y concibe el régimen político del discurso.

Sus indagaciones lo llevan a intentar explicar la manera como las relaciones de poder pueden penetrar materialmente en los cuerpos, sin tener que ser sustituidos por la representación de los sujetos. Foucault es suficientemente explícito al respecto:

... no soy de los que intentan estudiar los efectos de poder a nivel de la ideología. Me pregunto, en efecto, si antes de plantear la cuestión de la ideología, no sería más materialista estudiando la cuestión del cuerpo y los efectos de poder sobre él. ${ }^{23}$

Plantea que el poder no se ejerce a través de construcciones ideológicas, sino por medio de la red disciplinaria que consta de me22 Ibidem, p. 189.

23. "Poder-cuerpo" en Microfisica del poder, op. cit., p. 106. 
canismos sutiles que forman, organizan y ponen en circulación saberes. Las disciplinas disocian el cuerpo, ya que de una parte incrementan sus capacidades y fuerzas con miras a una mayor utilidad económica y de otra parte fabrican cuerpos sometidos, ejercitados y dóciles en términos políticos de obediencia. Concretamente, el éxito del poder disciplinario se debe a la inspección jerárquica, la sanción normalizadora y su combinación con el examen, que han producido al hombre del humanismo moderno. ${ }^{24}$

Desde esta perspectiva, el poder produce lo real a través de una transformación técnica de los individuos, y los mecanismos de represión e ideología no son más que estrategias extremas de poder. Foucault propone disminuir la imagen jurídica y negativa de las relaciones de poder, para concebir la preeminencia de su dimensión positiva y creativa. Y afirma, "lo que hace que el poder se sostenga, que sea aceptado, es simplemente que no pasa sólo como potencia que dice no, sino que produce cosas, induce placer, forma saber, produce discursos". 25

Puede apreciarse cómo, a diferencia del marxismo, no busca sujetos y objetos sino técnicas de producción y dominación. No se ocupa de la cosificación y enajenación de los hombres sino de investigar cómo el cuerpo es situado, marcado, temporalizado y coleccionado. Tampoco estudia el poder de la mente sobre la fuerza de trabajo, sino a la actividad corporal misma. Por tanto, para Foucault, la conciencia del cuerpo ha sido adquirida como resultado de la ocupación del cuerpo por el poder.

El seguimiento de la deconstrucción de la noción de ideología permite observar los procedimientos a través de los cuales el pensamiento foucaultiano logró sustituir la filosofía del objeto (objeto como fin o causa) por una filosofía de la relación (tomar el problema por la práctica o el discurso). Y, justamente, a partir de esta alternativa se hace posible avanzar aún más en el cuestionamiento del uso tradicional del término ideología. ${ }^{26}$

Desde esta perspectiva, la ideología idealiza las prácticas a través de racionalizaciones que pretenden explicarlas y se sirve de objetos naturales, dados e inmutables en su esencia, a través de la historia. Esto es, se ocupa de El Estado, El Poder, La Sociedad y

24 Vigilar y castigar, Siglo XXI editores, México, 1976, pp. 139-198.

25 "Verdad y poder", op. cit.

26. Paul|Veyne, Como se escribe la historia, Alianza Editorial, Madrid, 1984. 


\section{Sofía Gallardo}

demás, como fines o causas, en vez de referirse a las prácticas que los han constituido.

Foucault desplaza la noción de ideología para ocuparse de la descripción y explicación de lo que ha sido el "hacer" y el "decir" en cada momento de la historia. De esta manera, si prácticas son lo que hacen los hombres y el decir es en última instancia un hacer, no hay más que prácticas. Y si las prácticas son creaciones del azar de la historia y no de la conciencia, resulta claro que su propósito sea suprimir el término de ideología en su horizonte de racionalidad prometedora. 